\title{
Rubidium, zirconium, and lithium production in massive AGB stars
}

\author{
M.A. van Raai*i \\ Sterrekundig Instituut, University of Utrecht, Postbus 80000, 3508 TA Utrecht, The Netherlands \\ E-mail:m.a.vanraaiestudents.uu.nl
}

\section{Lugaro}

Sterrekundig Instituut, University of Utrecht, Postbus 80000, 3508 TA Utrecht, The Netherlands Centre for Stellar and Planetary Astrophysics, Monash University, Australia

\author{
A.I. Karakas \\ Research School of Astronomy and Astrophysics, Mt. Stromlo Observatory, Cotter Rd., Weston, \\ ACT Australia
}

\section{D.A. García-Hernández}

Instituto de Astrofisica de Canarias, La Laguna (Tenerife), España

\section{Yong}

Research School of Astronomy and Astrophysics, Mt. Stromlo Observatory, Cotter Rd., Weston, ACT Australia

A recent survey of a large sample of massive Galactic asymptotic giant branch (AGB) stars shows that significant overabundances of rubidium (up to 100 times solar) are present in these stars. Zirconium, on the other hand, is not enriched compared to the solar abundance. These observations can set constraints on our theoretical notion of the slow neutron capture process (the $s$ process) in AGB stars, as well as on the rates of the neutron capture reactions involved in the production of $\mathrm{Rb}$ and $\mathrm{Zr}$. Li abundances are also reported for these stars. Li is made via proton captures at the base of the convective envelope (hot bottom burning; HBB) in AGB models of mass higher than $\approx 5 M_{\odot}$. We present predictions of $\mathrm{Rb}, \mathrm{Zr}$, and Li abundances for AGB stars of masses 5 , 6 , and $6.5 M_{\odot}$. We find increasing Rb overabundances with increasing stellar mass, as observed, but we are far from matching the highest observed $\mathrm{Rb}$ enhancements, unless the third dredge up efficiency remains as high as before the onset of the superwind phase during the final few pulses of a massive AGB star. We predict large variations in the Li abundances, which are observed. Finally, we show that the Rb production increases with a decrease in the stellar metallicity, at a given mass. This result may be useful to constrain the source of abundance anomalies observed in Globular Cluster Stars.

10th Symposium on Nuclei in the Cosmos

July 27 - August 12008

Mackinac Island, Michigan, USA

\footnotetext{
*Speaker.

${ }^{\dagger} \mathrm{ML}$ is supported by NWO, AIK is supported by the ARC and received a grant from NWO and NOVA to visit Utrecht University during the time this work was prepared.
} 


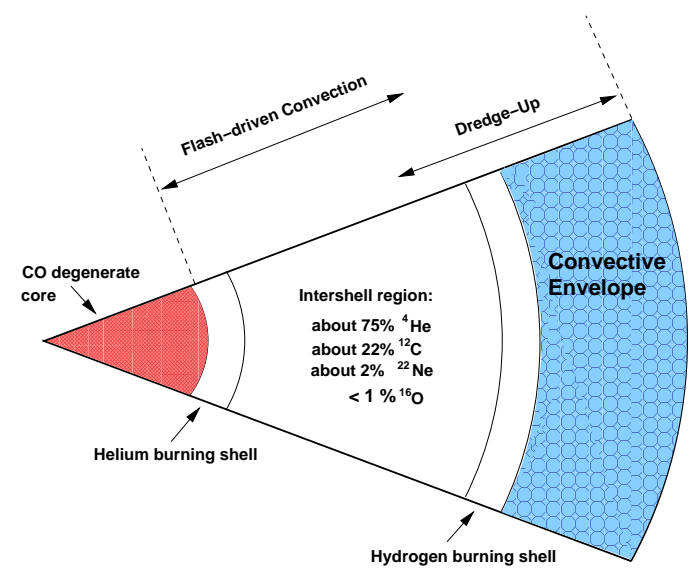

Figure 1: Schematic representation of the structure of an AGB star, from [1]

\section{Introduction}

Stars with an initial mass in the range $\approx 0.8$ to $8 M_{\odot}$ will evolve through the thermally-pulsing asymptotic giant branch (TP-AGB). Figure 1 shows a schematic representation of the structure of an AGB star. The H-burning shell is responsible for the nuclear energy production most of the time, but every $10^{4-5}$ years or so, the He shell ignites, driving convection throughout most of the He intershell, this is known as a thermal pulse (TP). The H-burning shell is temporarily shut off after a TP has occurred, because the energy released by the thermal pulse drives a strong expansion of the whole star. At this time the convective envelope extends inward and may reach the He-intershell; this is known as the third dredge-up (TDU) and can occur periodically after each TP. The TDU mixes nuclear burning products from the $\mathrm{He}$ and $\mathrm{H}$-burning shells to the stellar surface $[2,3]$. In the He intershell of AGB stars neutrons can be released by the ${ }^{13} \mathrm{C}(\alpha, n){ }^{16} \mathrm{O}$ reaction during the interpulse periods, and by the ${ }^{22} \mathrm{Ne}(\alpha, n)^{25} \mathrm{Mg}$ reaction in the convective TPs. Due to the availability of free neutrons, slow neutron captures (the $s$ process) can occur leading to the production of elements heavier than Fe (see, e.g., Ref. [4]). Massive AGB stars have initial masses between $\sim 4$ to $8 M_{\odot}$ and experience proton capture at the base of their convective envelope (hot bottom burning; HBB). ${ }^{7} \mathrm{Li}$ can be made by HBB during the interpulse through ${ }^{3} \mathrm{He}(\alpha, \gamma){ }^{7} \mathrm{Be}\left(e^{-} v\right){ }^{7} \mathrm{Li}$ via the Cameron-Fowler mechanism ([5]).

\section{The production of rubidium via the $s$ process}

Figure 2 shows a section of the chart of the nuclides from $\mathrm{Kr}$ to $\mathrm{Zr}$. When ${ }^{84} \mathrm{Kr}$ captures a neutron, $60 \%$ of the flux proceeds to the ground state of ${ }^{85} \mathrm{Kr}\left(\tau_{1 / 2}=3934.4\right.$ days $)$ and the other $40 \%$ proceeds to the metastable state of ${ }^{85} \mathrm{Kr}\left(\tau_{1 / 2}=4.480\right.$ hours $)$. The long decay time of the ${ }^{85} \mathrm{Kr}$ ground state allows it to capture another neutron, thus producing ${ }^{86} \mathrm{Kr}$ instead of ${ }^{85} \mathrm{Rb}$, provided the neutron density is higher than $\approx 5 \times 10^{8} \mathrm{~N}_{n} \mathrm{~cm}^{-3}$. ${ }^{86} \mathrm{Kr}$ can also capture a neutron producing ${ }^{87} \mathrm{Kr}$, which quickly decays into ${ }^{87} \mathrm{Rb}$. The other unstable isotope in Fig. 2 is ${ }^{86} \mathrm{Rb}\left(\tau_{1 / 2}=18.63\right.$ days). This branching point produces ${ }^{87} \mathrm{Rb}$ directly, with $\approx 50 \%$ of the flux going to ${ }^{87} \mathrm{Rb}$ if the neutron density is $\gtrsim 10^{10} \mathrm{~N}_{n} \mathrm{~cm}^{-3}$. 


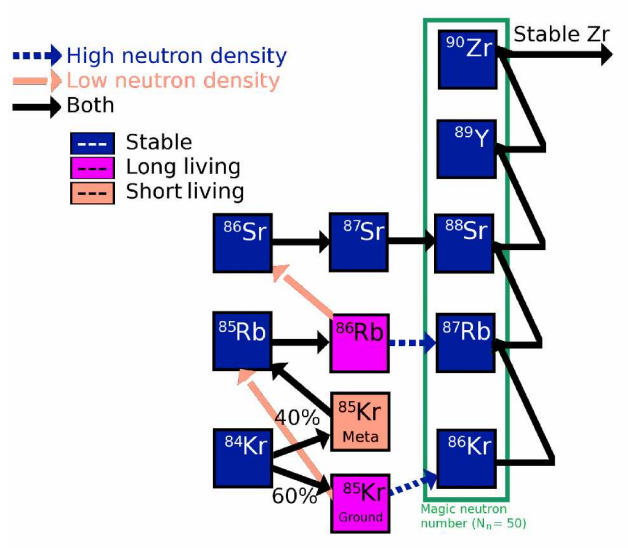

Figure 2: Section of the chart of the nuclides from $\mathrm{Kr}$ to $\mathrm{Zr}$. Stable isotopes are depicted with their designation in white on a dark background, unstable isotopes are depicted with their designation in black on a light background. The light arrows show the neutron capture flux for low neutron densities, the darker, dashed arrows for high neutron densities, and the black, solid arrows for both high and low neutron densities.

\begin{tabular}{ccc}
\hline & Low Mass $\left(1-3 M_{\odot}\right)$ & High Mass $\left(5-7 M_{\odot}\right)$ \\
\hline$[\mathrm{Rb} / \mathrm{Fe}]$ & -0.3 to 0.6 & 0.6 to 1.6 \\
{$[\mathrm{Zr} / \mathrm{Fe}]$} & $<1.0$ & $<0.5$ \\
{$[\mathrm{Rb} / \mathrm{Zr}]$} & $<0$ & 0.1 to 2.1 \\
\hline References & {$[6,7]$} & {$[8]$} \\
\hline
\end{tabular}

Table 1: Ranges of observed $\mathrm{Rb}$ and $\mathrm{Zr}$ abudances in AGB stars of different initial masses.

The maximum neutron density produced by the ${ }^{22} \mathrm{Ne}(\alpha, \mathrm{n})^{25} \mathrm{Mg}$ reaction in the thermal pulse of a $6.5 M_{\odot} Z=0.02$ model is about $10^{12-13} \mathrm{~N}_{n} \mathrm{~cm}^{-3}$, two orders of magnitude higher than in a $3 M_{\odot} Z=0.02$ model and over five orders of magnitude higher than the neutron density produced by the ${ }^{13} \mathrm{C}(\alpha, n){ }^{16} \mathrm{O}$ reaction. The ${ }^{22} \mathrm{Ne}(\alpha, \mathrm{n})^{25} \mathrm{Mg}$ reaction is believed to be the dominant neutron source in a massive AGB star and the ${ }^{13} \mathrm{C}(\alpha, n){ }^{16} \mathrm{O}$ reaction the dominant neutron source of a low mass AGB star. Thus, due to the activation of the branchings described above, a massive AGB star will produce a higher $\mathrm{Rb} / \mathrm{Zr}$ ratio than a low-mass AGB star (see Lugaro et al., these proceedings).

\section{Observations and models}

The survey by $[8,9]$ of massive Galactic AGB stars shows those stars have significant enhancements in rubidium, but not in zirconium. We summarize those observations in Table 1, where we also included the observational data for low-mass AGB stars by [6] and [7]. We discuss if we can quantitatively match these observations with our theoretical models. Since the observations are of Galactic-disk AGB stars, we focus on AGB models of solar metallicity. The initial abundances we use were taken from [10] for $Z=0.02$. We calculated models with masses 5, 6, and $6.5 M_{\odot}$. We also calculated two models of a $5 M_{\odot}$ star at $Z=0.008$ and $Z=0.004$ where we used scaled 


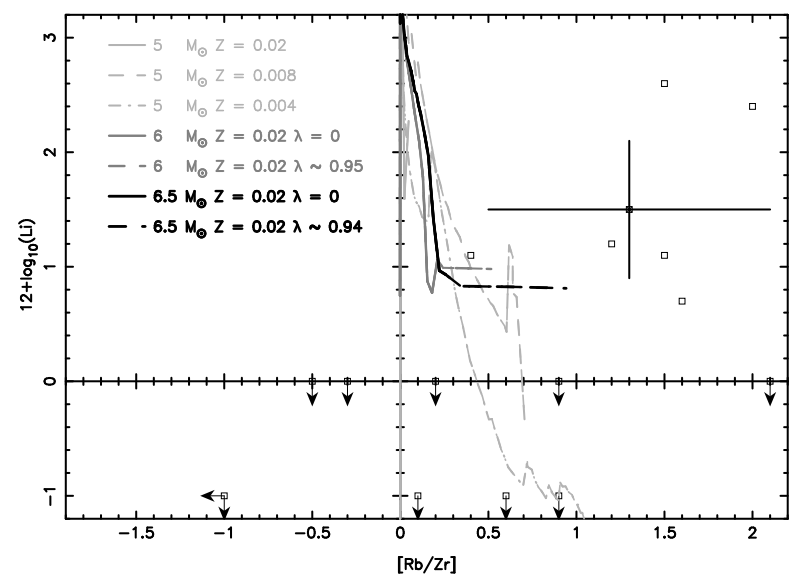

Figure 3: Results of synthetic extended evolution (see text) and models of different metallicity (the $5 M_{\odot}$ models). The black squares are observations, the lines are theoretical predictions. The errorbars are maximum errors for each individual data point. Arrows indicate upper limits. The solid part of the 6 and 6.5 $M_{\odot}$ model lines are calculated without the synthetic extension and are equivalent to a TDU efficiency of 0 during the extended evolution. The dashed parts of those lines are the result of the synthetic extension when the TDU efficiency is chosen to remain constant during the synthetic extension.

abundances of [10]. As a consistency check we also calculated models of $3 M_{\odot}$ to see if we could match the data for low-mass stars by [6] and [7] (see Table 1).

\section{Results}

We predict a $[\mathrm{Rb} / \mathrm{Zr}]$ down to $0.5,[\mathrm{Rb} / \mathrm{Fe}]$ up to 0.5 , and a $[\mathrm{Zr} / \mathrm{Fe}]$ up to 1.0 for a $3 M_{\odot}$ AGB model, depending on the amount of ${ }^{13} \mathrm{C}$ available in the He intershell for the ${ }^{13} \mathrm{C}(\alpha, n){ }^{16} \mathrm{O}$ reaction. These values are in agreement with the observations.

Figure 3 shows the observations together with our theoretical predictions for the massive AGB stars. Due to convergence problems, our evolution models can only calculate the AGB phase up to the point where significant mass loss occurs. The TDU efficiency is very uncertain for small envelope masses. We have estimated the effect of the remaining TPs on the $[\mathrm{Rb} / \mathrm{Fe}] \mathrm{ratio}$, assuming a maximum TDU efficiency (dashed part of the lines). The stars are observed at the final stages of the AGB, so, to match the observed data one should look at the end of each prediction line. The $[\mathrm{Rb} / \mathrm{Fe}]$ ratio increases with increasing mass, as observed (see Fig. 2 of [8]). Our most massive solar metallicity model has a $[\mathrm{Rb} / \mathrm{Fe}]$ abundance of $\sim 1$, which is a good match to the observed abundances, given the large errors.

The lightest lines in fig. 3 show the results for a $5 M_{\odot}$ model at metallicities of $Z=0.02, Z$ $=0.008$, and $Z=0.004$. These models were not extended using the synthetic algorithm described above. The $[\mathrm{Rb} / \mathrm{Fe}]$ ratio increases with decreasing metallicity, this result may be useful to constrain the source of abundance anomalies observed in Globular Cluster Stars ([11]).

A wide range of Li abundances can be seen in both models and observations. We do not take into account the effects of HBB and Li production on the surface abundances, with the synthetic algorithm. Further investigation of the Li abundances is necessary to see if we can quantitatively 
match all observed Li abundances (note that the low metallicity models should not be used to match the observed Li abundances, as the observations are of solar metallicity stars).

\section{Conclusions}

We predict $[\mathrm{Rb} / \mathrm{Zr}]>0$ for massive AGB stars, as well as the observed increase with increasing stellar mass. However, even if we take our most massive model with maximum TDU efficiency during the final synthetic pulses, we cannot match the highest $[\mathrm{Rb} / \mathrm{Fe}]$ abundances observed. These may be even more massive AGB stars, for which we do not yet have models. A better understanding of the TDU efficiency with receding envelope mass is essential for further investigation of this issue, as well as more precise determination of spectroscopic abundances. We find that the $[\mathrm{Rb} / \mathrm{Fe}]$ ratio increases with a decrease in the stellar metallicity, at a given mass. This result may be useful to constrain the source of abundance anomalies observed in Globular Cluster Stars. Further analysis of the Li abundances is necessary to see if we can quantitatively match all observed $\mathrm{Li}$ abundances.

\section{References}

[1] Karakas, A. I., Lattanzio, J. C., and Pols, O. R. 2002, Parameterising the Third Dredge-up in Asymptotic Giant Branch Stars, PASA 19, 515-526.

[2] Herwig, F. 2004, Dredge-up and Envelope Burning in Intermediate-Mass Giants of Very Low Metallicity, ApJ 605, 425-435.

[3] Karakas, A., and Lattanzio, J. C. 2007, Stellar Models and Yields of Asymptotic Giant Branch Stars, Publications of the Astronomical Society of Australia 24, 103-117.

[4] Gallino, R., Arlandini, C., Busso, M., Lugaro, M., Travaglio, C., Straniero, O., Chieffi, A., and Limongi, M. 1998, Evolution and Nucleosynthesis in Low-Mass Asymptotic Giant Branch Stars. II. Neutron Capture and the s-Process, ApJ 497, 388.

[5] Cameron, A. G. W., and Fowler, W. A. 1971, Lithium and the s-PROCESS in Red-Giant Stars, ApJ 164, 111-114.

[6] Abia, C., Busso, M., Gallino, R., Domínguez, I., Straniero, O., and Isern, J. 2001, The ${ }^{85}$ Kr s-Process Branching and the Mass of Carbon Stars, ApJ 559, 1117-1134.

[7] Lambert, D. L., Smith, V. V., Busso, M., Gallino, R., and Straniero, O. 1995, The Chemical Composition of Red Giants. IV. The Neutron Density at the s-Process Site, ApJ 450, 302-+.

[8] García-Hernández, D. A., García-Lario, P., Plez, B., D’Antona, F., Manchado, A., and Trigo-Rodriguez, J. M. 2006, Rubidium-Rich Asymptotic Giant Branch Stars, Science 314, 1751.

[9] García-Hernández, D. A., García-Lario, P., Plez, B., Manchado, A., D’Antona, F., Lub, J., and Habing, H. 2007, Lithium and zirconium abundances in massive Galactic $O$-rich AGB stars, $A \& A$ 462, 711-730.

[10] Anders, E., and Grevesse, N. 1989, Abundances of the elements - Meteoritic and solar, Geochim. Cosmochim. Acta 53, 197-214.

[11] Yong, D., Aoki, W., Lambert, D. L., and Paulson, D. B. 2006, Rubidium and Lead Abundances in Giant Stars of the Globular Clusters M13 and NGC 6752, ApJ 639, 918-928. 